\title{
SLC12A2 Gene
}

National Cancer Institute

\section{Source}

National Cancer Institute. SLC12A2 Gene. NCI Thesaurus. Code C102513.

This gene is involved in ion transport. 